\section{Efficacy of Metal Artifact Reduction Algorithm of Cone-Beam Computed Tomography for Detection of Fenestration and Dehiscence around Dental Implants}

\author{
Fatemeh Salemi'*®, Mohamad Reza Jamalpour², Amir \\ Eskandarloo', Leili Tapak³ ${ }^{3}$ Narges Rahimi ${ }^{4}$
}

\begin{abstract}
Background: Beam hardening and scattering artifacts from high-density objects such as dental implants adversely affect the image quality and subsequently the detection of fenestration or dehiscence around dental implants.

Objective: This study aimed to assess the efficacy of metal artifact reduction (MAR) algorithm of two cone-beam computed tomography (CBCT) systems for detection of peri-implant fenestration and dehiscence.

Material and Methods: In this experimental study, thirty-six titanium implants were placed in bone blocks of bovine ribs. Fenestration and dehiscence were created in the buccal bone around implants. CBCT images were obtained using Cranex 3D and ProMax 3D CBCT systems with and without MAR algorithm. Two experienced radiologists observed the images. Data were analyzed using SPSS software. The Kappa coefficient of agreement, the area under the receiver operating characteristic (ROC) curve, sensitivity, specificity, and accuracy of different imaging modalities were calculated and analyzed.
\end{abstract}

Results: In both CBCT systems, the use of MAR algorithm decreased the area under the ROC curve and subsequently the diagnostic accuracy for the detection of fenestration and dehiscence. The sensitivity, specificity and accuracy of both CBCT systems were higher in absence of the MAR algorithm. The specificity of ProMax 3D for detection of fenestration was equal with/without the MAR algorithm.

Conclusion: Although CBCT is suitable for detection of peri-implant defects, the application of the MAR algorithm does not enhance the detection of peri-implant fenestration and dehiscence.

Citation: Salemi F, Jamalpour MR, Eskandarloo A, Tapak L, Rahimi N. Efficacy of Metal Artifact Reduction Algorithm of Cone-Beam Computed Tomography for Detection of Fenestration and Dehiscence around Dental Implants. J Biomed Phys Eng. 2021;11(3):305-314. doi: $10.31661 /$ jbpe.v0i0.2102-1284.

\section{Keywords}

Cone-Beam Computed Tomography; Metal Artifact Reduction Algorithm; Fenestration; Dehiscence; Dental Implants; ROC Curve

\section{Introduction}

$\mathrm{T}$ he long-term success of implant treatment mainly depends on the health of peri-implant soft and hard tissues. Absence of pain, tenderness, mobility, inflammation and infection often indicates a successful implant treatment. Also, peri-implant marginal bone loss should not exceed 1-2 $\mathrm{mm}$ in the first year after treatment and the acceptable level of bone loss in the next years is $0.2 \mathrm{~mm}$ [1]. Presence
${ }^{1} \mathrm{PhD}$, Department of Oral \& Maxillofacial

Radiology, School of

Dentistry, Hamadan

university of Medical Sci-

ence, Hamadan, Iran

${ }^{2} \mathrm{MScD}$, Department of

Oral and Maxillofacial

Surgery, Dental Implants

Research Center, School

of Dentistry, Hamadan

university of Medical Sci-

ence. Hamadan, Iran

${ }^{3} \mathrm{PhD}$, Department of

Biostatistics, School of

Health Modeling of Non-

communicable Diseases

Research Center, Health

Sciences \& Technol-

ogy Research Institute,

Hamadan University

of Medical Sciences,

Hamadan, Iran

${ }^{4}$ DMD, General Dentist,

Hamadan University

of Medical Sciences,

Hamadan, Iran

*Corresponding author:

Fatemeh Salemi

Department of Oral and

Maxillofacial Radiology,

School of Dentistry,

Hamadan University

of Medical Sciences,

Hamadan, Iran

E-mail: dr.salemi@yahoo.

com

Received: 16 February 2021

Accepted: 2 April 2021 
of adequate peri-implant bone is another factor, contributing to the success of implant treatment. Inadequate bone volume in buccolingual direction may cause fenestration and dehiscence and compromise the long-term prognosis of implant [2].

Fenestration is a local defect that involves part of the peri-implant bone surface without the bone margin. Dehiscence is a local bone defect, extending from the cervical part of implant towards the apical [3]. Fenestration and dehiscence can compromise the peri-implant bone support and lead to peri-implantitis and failure of osseointegration. Early detection of these defects can help prevent subsequent complications and increase the long-term survival of implants [4].

Radiographic assessment of bone after implant placement is highly important and is often performed by intraoral radiography. However, conventional intraoral radiographs only visualize the mesial and distal parts of the alveolar bone and have limitations in visualization of buccal and lingual bone defects due to the superimposition of anatomical structures [5].

Cone-beam computed tomography (CBCT) provides three-dimensional images of periimplant cortical bone and enables the observation of buccal and lingual cortices [4]. Also, CBCT can be used for assessment of the marginal contour of bone and detection of interosseous defects such as fenestration and dehiscence, which cannot be visualized by the conventional radiography [6]. Despite the numerous advantages of CBCT over the conventional two-dimensional radiographic modalities, the quality of CBCT images is affected by artifacts. In oral cavity artifacts caused by high-density objects such as dental implants, amalgam restorations and root filling materials account for the majority of $\mathrm{CBCT}$ scan artifacts. These artifacts are basically called beam hardening and can decrease the diagnostic value of CBCT [7]. When the X-ray beam passes through a high-density object, low-energy photons are absorbed by a greater amount than high-energy photons. Beam hardening creates two types of artifacts as follows: 1) artifacts related to metal structures, known as cupping artifacts, and 2) streaks and bands, creating extinction or missing value artifacts between two dense objects [8]. These artifacts can be mistaken for pathologies or mask the anatomical structures and complicate correct diagnosis [9]. Two CBCT artifact reduction strategies have been proposed in the literature. The first one is to adjust the exposure settings by increasing the amperage $(\mathrm{mA})$, time and voltage $(\mathrm{kVp})$. The other strategy is to use metal artifact reduction (MAR) algorithms during image reconstruction to improve the image quality $[7,10]$.

Considering the fact that artifacts caused by dental implants can affect radiographic assessment of peri-implant bone defects, this study aimed to assess the efficacy of MAR algorithm of two CBCT systems for detection of periimplant fenestration and dehiscence.

\section{Material and Methods}

This experimental study, approved by the research ethics committee of Hamadan University of Medical Science (ethics number: IR.UMSHA.REC.1396.482), used the rib bones of freshly slaughtered cows, due to their high resemblance to alveolar bone. The ribs were refrigerated to prevent their dehydration. The soft tissue around the ribs was removed and the rib bones were cut into 36 bone blocks with precisely equal dimensions, measuring $8 \times 8 \mathrm{~mm}$ with $11 \mathrm{~mm}$ height, using a CNC machine (Pooya Mekatronic Knowledg based company, Hamadn, Iran) [10]. Also, a plastic box was designed by the same manufacturer (Pooya Meatronic, Iran) with the exact same size as the bone blocks, firmly placed in the respective plastic box for implant insertion (Figure 1). Moreover, 36 titanium implants (SIC invent AG, Switzerland) with $4 \mathrm{~mm}$ diameter and $11 \mathrm{~mm}$ length were inserted in bone blocks by an experienced oral and max- 
illofacial surgeon (Figure 2). Considering the size of implants and bone blocks, the distance between the implants and the reference buccal surface was $2 \mathrm{~mm}$.

Boxes in the shape and form of a mandible were fabricated using five layers of red dental wax with $2 \mathrm{~mm}$ thickness, and the bone blocks were placed in these boxes (Figure 3 ). The control CBCT images were obtained from the bone blocks with inserted implants prior to the creation of defects using Cranex 3D and ProMax 3D CBCT systems with and without enabling the MAR algorithm. Spherical and cylindrical dental burs were used to create

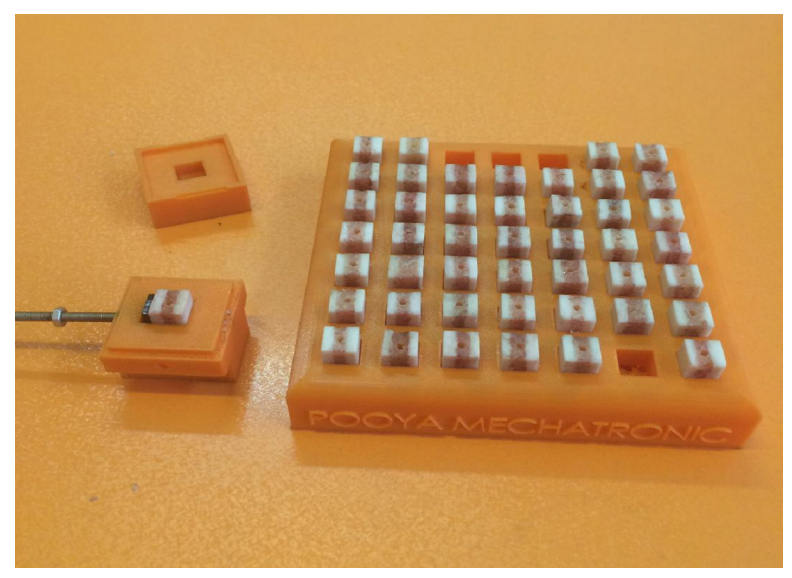

Figure 1: Bone blocks were prepared with equal dimensions, measuring $8 \times 8 \mathrm{~mm}$ with $11 \mathrm{~mm}$ height and plastic box was designed.
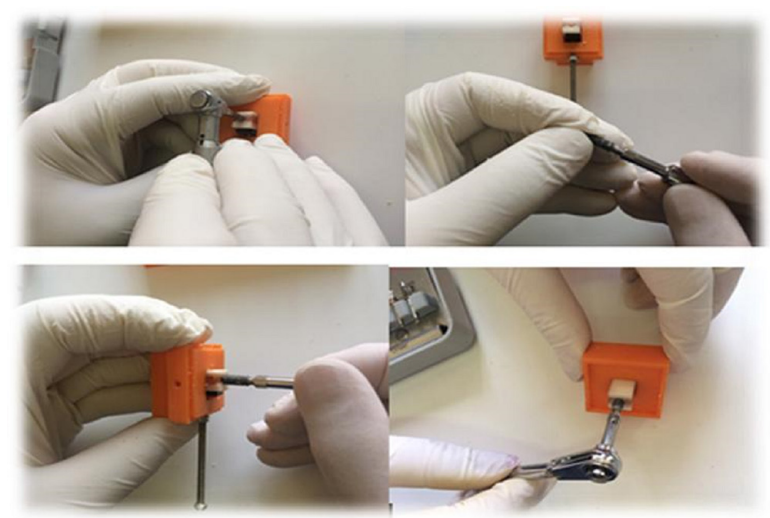

Figure 2: The bone block was firmly placed in the plastic box and implants were inserted in bone block.

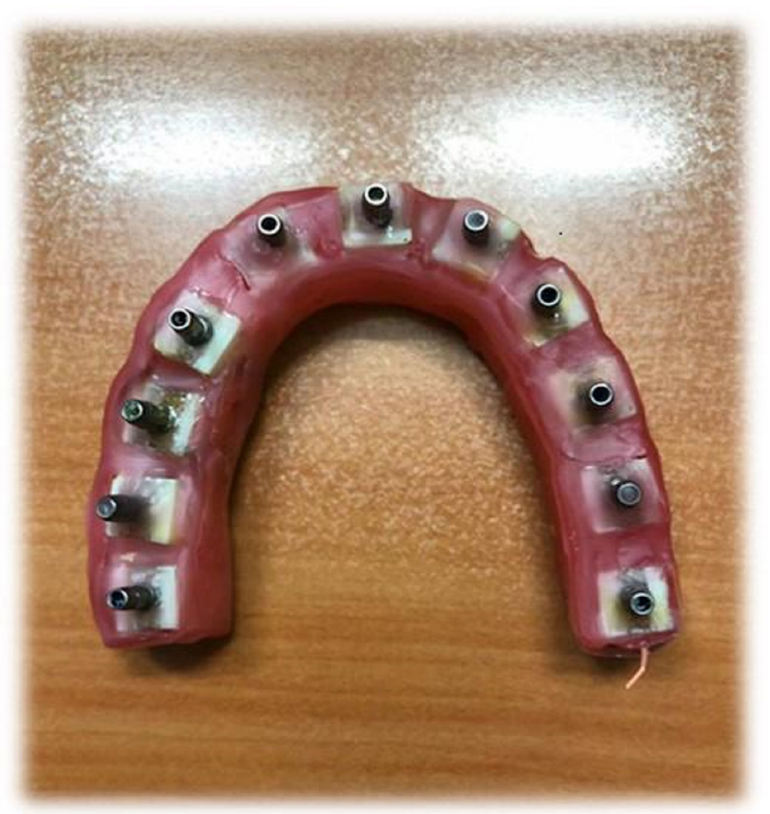

Figure 3: The boxes were fabricated in the shape and form of a mandible using five layers of red dental wax.

dehiscence and fenestration defects. The dehiscence defect was created at the bone block margin and cervical part of the implant in a semilunar shape with $2 \mathrm{~mm}$ diameter. The fenestration defect was created $10 \mathrm{~mm}$ lower than the bone margin at the apical region of the implant with $2 \mathrm{~mm}$ diameter. In order to better simulate the natural defects, the margin of created defects were beveled. A digital caliper (Mitutoyo Corp, Kawasaki, Japan) was used to measure the depth and width of defects. The bone blocks were then placed again in wax boxes and underwent CBCT [1]. CBCT images were obtained using ProMax 3D CBCT system (Planmeca, Helsinki, Finland) with $200 \mu$ voxel size and $7.5 \times 11 \mathrm{~cm}^{2}$ the field of view, with the exposure settings of $84 \mathrm{kVp}$ and $14 \mathrm{~mA}$ and $12 \mathrm{~s}$ time, and Cranex 3D (Soredex, Helsinki, Finland) with $200 \mu$ voxel size, $6 \times 8 \mathrm{~cm}^{2}$ field of view, $90 \mathrm{kVp}, 10 \mathrm{~mA}$ and $14 \mathrm{~s}$ time.

For all CBCT scans, the wax rim was located in the same reproducible position in the system. CBCT scans were obtained twice with 
and without enabling the MAR algorithm. The ProMax 3D images were saved in Romexis software version 2.9.2 (Planmeca, Helsinki, Finland) while the Cranex 3D images were saved in On Demand software (Cybermed Inc., Seoul, Korea). For assessment of images, five cross-sections with $1 \mathrm{~mm}$ slice thickness and $1 \mathrm{~mm}$ interval were reconstructed such that one of the sections passed through the implant center (Figures 4 and 5).

Two oral and maxillofacial radiologists evaluated all the images twice with two week interval and expressed their opinion regarding the presence/absence of fenestration and dehiscence defects and recorded the results in a checklist. The observers were free to assess the images in coronal, axial and sagittal planes. They were also allowed to adjust the brightness, contrast and magnification of im-
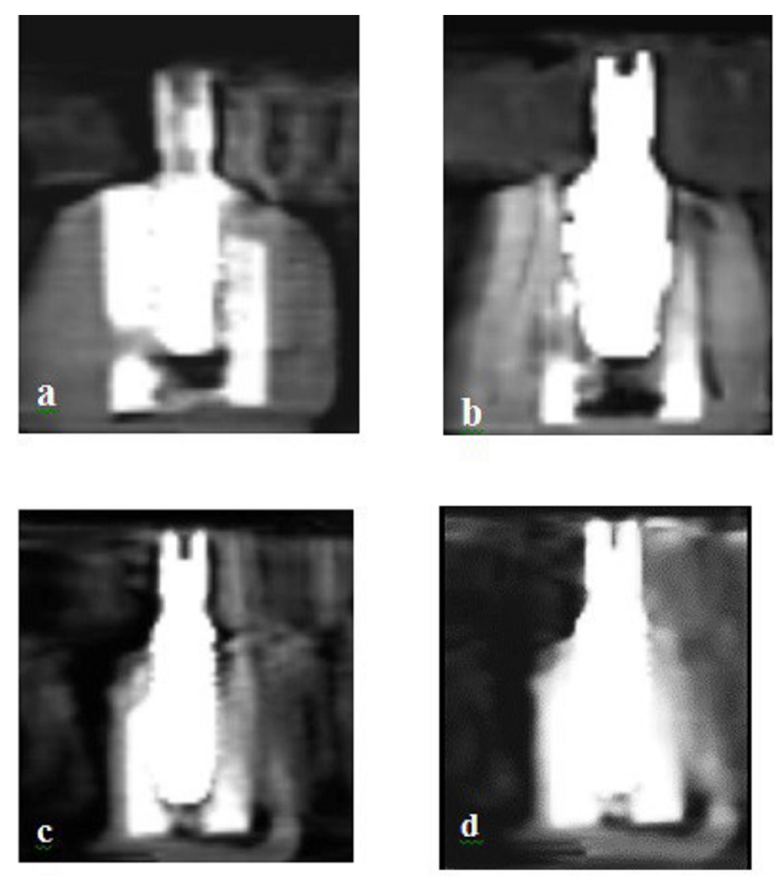

Figure 4: Cone Beam Computed Tomography (CBCT) images were obtained using ProMax 3D CBCT systema a. Fenestration without Metal Artifact Reduction b. Fenestration with Metal Artifact Reduction c. Dehiscence without Metal Artifact Reduction d. Dehiscence with Metal Artifact Reduction. ages.

Data were analyzed using SPSS software version 22. The data were reported as mean and standard deviation. The area under the receiver operating characteristic curve (ROC), sensitivity, specificity, positive predictive value (PPV) and negative predictive value (NPV) were calculated and reported for different imaging modalities. Comparisons were made based on independent t-test. The kappa statistics was used to determine the intra- and inter observer agreements.

\section{Results}

The intraobserver agreement was moderate for images obtained with the MAR algorithm and good to excellent for images obtained without the MAR algorithm. The interobserver agreement was poor to moderate for images obtained with the MAR algorithm and good to excellent for images obtained without this algorithm (Table 1).

The area under the ROC curve was calculated for images of both CBCT systems taken with and without the MAR algorithm separately for the fenestration, dehiscence and nodefect groups (Table 2).

A: With the MAR algorithm; B: Without the MAR algorithm; SD: Std. deviation.

Considering the ROC values, the use of MAR algorithm decreased the diagnostic accuracy (a reduction in area under the ROC curve) for detection of both defects; this reduction was statistically significant for fenestration $(\mathrm{P}=0.020)$ and no-defect group $(\mathrm{P}=0.011)$. However, it was not significant for dehiscence $(\mathrm{P}=0.204)$ in ProMax 3D. Regarding the Cranex 3D images, the reduction in accuracy was not significant for fenestration $(\mathrm{P}=0.0798)$, but it was statistically significant for dehiscence $(\mathrm{P}=0.004)$ and no-defect group $(\mathrm{P}=0.001)$.

The sensitivity, specificity, PPV, NPV and accuracy of each imaging modality were calculated separately for fenestration and dehiscence defects (Table 3). For the ProMax 3D 

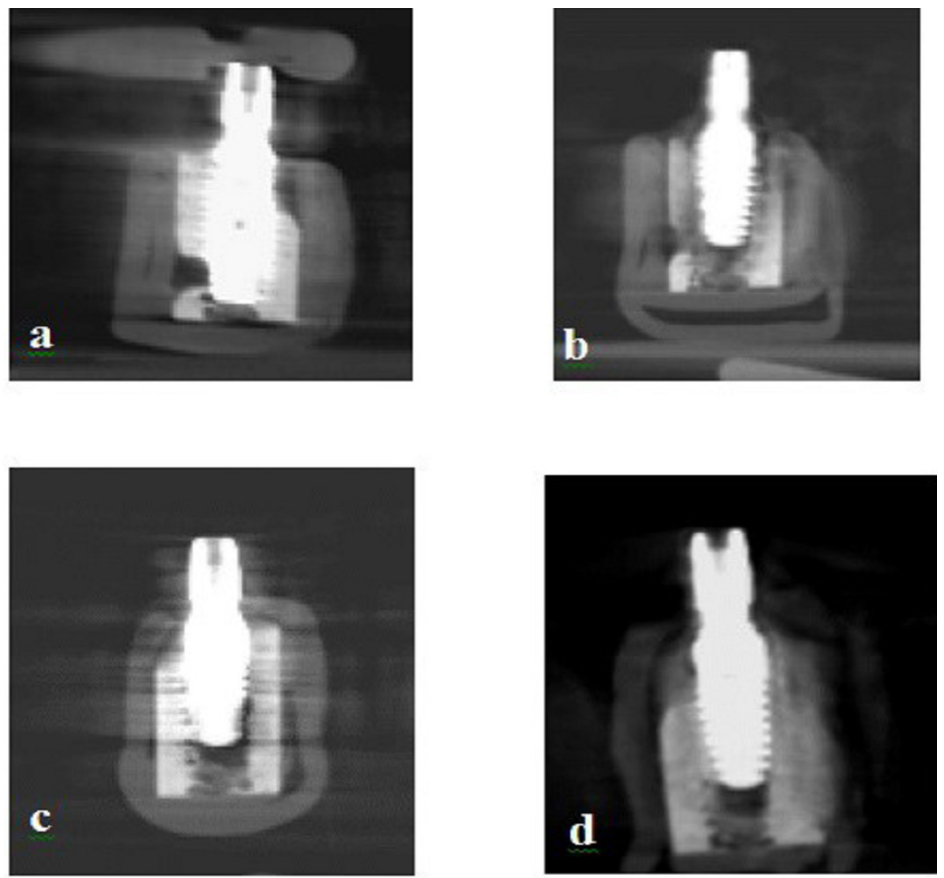

Figure 5: Cone Beam Computed Tomography (CBCT) images were obtained using Cranex 3D CBCT systema a. Fenestration without Metal Artifact Reduction b. Fenestration with Metal Artifact Reduction c. Dehiscence without Metal Artifact Reduction d. Dehiscence with Metal Artifact Reduction.

Table 1: Kappa values (mean and std. deviation) for the intra- and interobserver agreements

\begin{tabular}{ccccccc} 
Technique & \multicolumn{2}{c}{ Fenestration } & \multicolumn{2}{c}{ dehiscence } & \multicolumn{2}{c}{ No defect } \\
\hline & Intraobserver & Interobserver & Intraobserver & Interobserver & Intraobserver & Interobserver \\
\hline${ }^{*}$ A & $0.692(0.175)$ & $0.660(0.131)$ & $0.510(0.19)$ & $0.442(0.213)$ & $0.442(0.55)$ & $0.348(0.60)$ \\
\hline${ }^{* *}$ B & $0.948(0.74)$ & $0.948(0.37)$ & $0.813(0.265)$ & $0.771(0.218)$ & $0.771(0.211)$ & $0.714(0.202)$
\end{tabular}

*With the Metal Artifact Reduction algorithm; **Without the Metal Artifact Reduction algorithm

images, all these values for detection of the no-defect control group and dehiscence in absence of the MAR algorithm were higher than the corresponding values in the presence of the MAR algorithm. For detection of fenestration, the sensitivity, accuracy and NPV were higher in absence of the MAR algorithm, but the specificity and PPV were the same in presence and absence of the MAR algorithm in ProMax 3D images.

In Cranex 3D images, all these values for the detection of no-defect control group, dehiscence and fenestration in absence of the MAR algorithm were higher than the corresponding values in the presence of the MAR algorithm.

\section{Discussion}

Replacement of the lost teeth with dental implants is performed to restore optimal esthetics and function with no adverse effects on the adjacent soft and hard tissues. Success of implant treatment depends on many factors, including the quality and quantity of bone. Radiographic modalities are highly important for assessment of peri-implant bone. Periapical radiography with the parallel technique is often 
Table 2: Area under the Receiver Operating Characteristic (ROC) curve for images of both Cone Beam Computed Tomography systems taken with and without the Metal Artifact Reduction algorithm for the two observers.

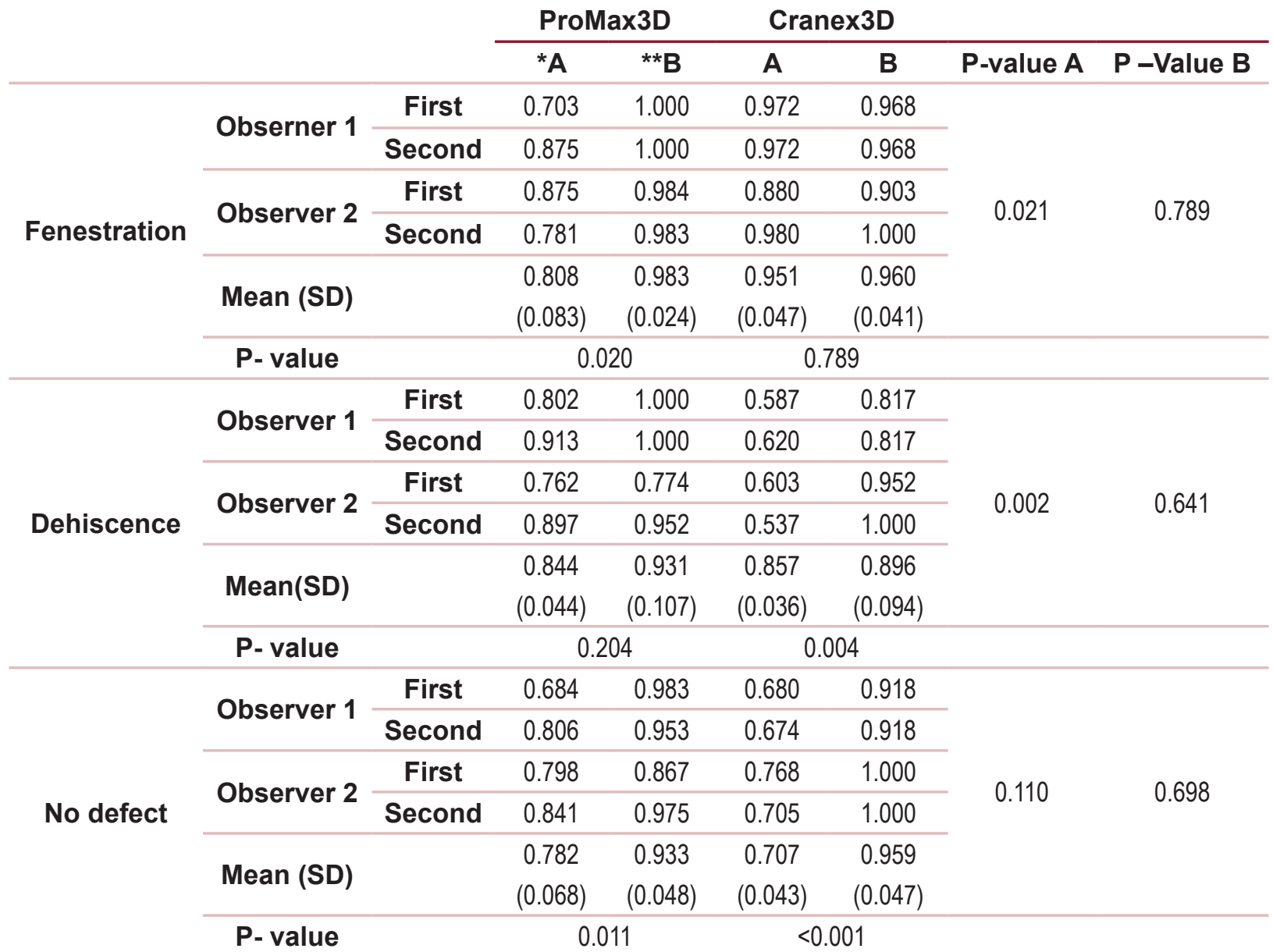

*With the Metal Artifact Reduction algorithm; **Without the Metal Artifact Reduction algorithm

used for postoperative assessment, following dental implant placement because it is suitable for assessment of the quality of osseointegration and presence of peri-implant inflammation. Periapical radiography is also used to assess the level of interproximal bone around dental implants [11]. However, periapical radiography has some limitations, providing a two-dimensional image of a three-dimensional structure. Also, the superimposition of the adjacent structures complicates adequate detection of peri-implant defects. On the other hand, it does not enable the precise assessment of the buccal and lingual bone plates. Thus, in case of suspecting the presence of fenestration or dehiscence around dental implants,
CBCT should be considered as an alternative imaging modality [12]. CBCT enables precise measurement of the cortical bone thickness around dental implants and is suitable for measurement of the dimensions of peri-implant defects, and also provides images with much higher quality compared with periapical and panoramic radiography [13]. When highdensity objects such as dental implants are visualized on CBCT scans, beam hardening and scattering artifacts adversely affect the image quality and subsequently the detection of fenestration or dehiscence around dental implants $[2,14]$.

Several strategies have been proposed for CBCT artifact reduction such as the use of a 
Efficacy of Metal Artifact Reduction Algorithm

Table 3: Sensitivity, specificity, Positive Predictive Value, Negative Predictive Value and accuracy of ProMax 3D and Cranex 3D Cone BeamComputed Tomography systems with and without the Metal Artifact Reduction algorithm for detection of dehiscence, fenestration and no-defect control group.

\begin{tabular}{|c|c|c|c|c|c|c|c|}
\hline & & $\begin{array}{c}\text { Dehiscence } \\
\text { Mean } \\
(95 \% \mathrm{Cl})\end{array}$ & $\begin{array}{l}\text { Fenestration } \\
\text { Mean }(95 \% \mathrm{Cl})\end{array}$ & $\begin{array}{l}\text { Control } \\
\text { Mean } \\
(95 \% \mathrm{Cl})\end{array}$ & $\begin{array}{l}\text { Dehiscence } \\
\text { Mean }(95 \% \mathrm{Cl})\end{array}$ & $\begin{array}{l}\text { Fenestration } \\
\text { Mean }(95 \% \mathrm{Cl})\end{array}$ & $\begin{array}{l}\text { Control } \\
\text { Mean } \\
(95 \% \mathrm{Cl})\end{array}$ \\
\hline \multirow{2}{*}{ Sensitivity } & ${ }^{*} \mathrm{~A}$ & $0.833(0.645,1)$ & $\begin{array}{c}0.617 \\
(0.476,0.758)\end{array}$ & $\begin{array}{c}0.758 \\
(0.574,0.941)\end{array}$ & $\begin{array}{c}0.701 \\
(0.488,0.914)\end{array}$ & $0.91(0.825,0.995)$ & $\begin{array}{c}0.805 \\
(0.646,0.963)\end{array}$ \\
\hline & ${ }^{* *} \mathrm{~B}$ & $0.862(0.652,1)$ & $0.966(0.924,1)$ & $0.945(0.882)$ & $0.833(0.645,1)$ & $\begin{array}{c}0.919 \\
(0.849,0.988)\end{array}$ & 1 \\
\hline \multirow{2}{*}{ Specificity } & $A$ & $0.853(0.687,1)$ & 1 & $0.848(0.695,1)$ & $\begin{array}{c}0.868 \\
(0.798,0.938)\end{array}$ & $\begin{array}{c}0.992 \\
(0.984,0.999)\end{array}$ & $\begin{array}{c}0.606 \\
(0.527,0.685)\end{array}$ \\
\hline & B & 1 & 1 & $0.920(0.795,1)$ & $\begin{array}{c}0.960 \\
(0.921,0.999)\end{array}$ & 1 & $0.918(0.825,1)$ \\
\hline \multirow{2}{*}{ ***PPV } & $A$ & $\begin{array}{c}0.884 \\
(0.802,0.967)\end{array}$ & 1 & $\begin{array}{c}0.772 \\
(0.598,0.945)\end{array}$ & $\begin{array}{c}0.567 \\
(0.461,0.673)\end{array}$ & $\begin{array}{c}0.980 \\
(0.960,0.999)\end{array}$ & $\begin{array}{c}0.540 \\
(0.525,0.554)\end{array}$ \\
\hline & B & 1 & 1 & $0.895(0.740,1)$ & $\begin{array}{c}0.913 \\
(0.546,0.980)\end{array}$ & 1 & $0.881(0.746,1)$ \\
\hline \multirow[t]{2}{*}{${ }^{\star * * *}$ NPV } & $A$ & $0.934(0.867,1)$ & $\begin{array}{c}0.819 \\
(0.774,0.864)\end{array}$ & $\begin{array}{c}0.869 \\
(0.779,0.960)\end{array}$ & $\begin{array}{c}0.699 \\
(0.677,0.721)\end{array}$ & $\begin{array}{c}0.966 \\
(0.936,0.996)\end{array}$ & $\begin{array}{c}0.861 \\
(0.759,0.963)\end{array}$ \\
\hline & $\mathrm{B}$ & $0.941(0.854,1)$ & $0.984(0.967,1)$ & $0.972(0.941,1)$ & $0.930(0.849,1)$ & $0.970(0.935,1)$ & 1 \\
\hline \multirow{2}{*}{ Accuracy } & $A$ & $\begin{array}{c}0.847 \\
(0.759,0.934)\end{array}$ & $\begin{array}{c}0.864 \\
(0.814,0.913)\end{array}$ & $\begin{array}{c}0.808 \\
(0.763,0.853)\end{array}$ & $\begin{array}{c}0.669 \\
(0.644,0.694)\end{array}$ & $\begin{array}{c}0.968 \\
(0.947,0.990)\end{array}$ & $\begin{array}{c}0.678 \\
(0.659,0.696)\end{array}$ \\
\hline & B & $0.953(0.882,1)$ & $0.992(0.978,1)$ & $\begin{array}{c}0.902 \\
(0.833,0.971)\end{array}$ & $\begin{array}{c}0.892 \\
(0.793,0.991)\end{array}$ & $\begin{array}{c}0.973 \\
(0.949,0.996)\end{array}$ & $0.946(0.884,1)$ \\
\hline
\end{tabular}

*With the MAR algorithm; **Without the MAR algorithm *** Positive Predictive Value **** Negative Predictive Value

small size field of view, anti-scatter grids and adjustment of exposure factors, with variable degrees of success [15]. Moreover, the MAR algorithms recently introduced by a number of manufacturers have greatly enhanced the quality of images [10].

In the present study, the kappa statistics showed that when the MAR algorithm was used, inter and intraobserver agreements decreased. This finding indicates that the MAR algorithm did not improve the performance of the observers. On the other hand, inter- and intraobserver agreements were higher for the detection of fenestration than dehiscence defects, irrespective of the imaging modality and the MAR mode. This finding was in agreement with that of Azevedo-Vaz et al. [1].

According to the literature, the area under the ROC curve or the AUC value indicates the technique accuracy [2]. The current results indicated that for the ProMax 3D images, the area under the ROC curve for fenestration was 0.983 without the MAR and 0.808 with the MAR algorithm. These values for dehiscence were 0.931 without the MAR and 0.844 with the MAR algorithm. In the control group, the area under the curve was 0.933 without the MAR and 0.782 with the MAR algorithm. For the Cranex 3D images, the area under the curve for fenestration was 0.960 without the MAR and 0.951 with the MAR algorithm. For dehiscence, the value was 0.896 without the MAR and 0.857 with the MAR algorithm. The area under the curve was 0.959 without the MAR and 0.707 with the MAR algorithm, respectively, for the control group. These find- 
ings indicate that in general, the area under the curve and subsequently the diagnostic accuracy for detection of fenestration was higher than that for the detection of dehiscence that was in accordance with the results of Sheikhi et al. [16] and Azevedo-Vaz et al. [2]. According to the study by Azevedo-Vaz et al. [2], the detection of a dehiscence defect with only one inferior border is more difficult than detection of a fenestration defect with one superior and one inferior border [2]. In the present study, in both ProMax 3D and Cranex 3D CBCT systems, the use of MAR algorithm decreased the area under the curve and subsequently the diagnostic accuracy. These results were in agreement with those of Azevedo-Vaz et al. [2] and Kamburoglu et al. [4].

Azevedo-Vaz et al. [2] evaluated the effect of MAR algorithm and the voxel size on diagnostic accuracy and found no significant difference between 0.2 and $0.3 \mathrm{~mm}$ voxel sizes for detection of fenestration and dehiscence. Since a larger voxel size decreases the patient radiation dose, the use of a larger voxel size for the assessment of peri-implant cortical bone is recommended [2]. In another study, AzevedoVaz et al. [2] concluded that although voxel size does not affect the diagnostic accuracy for detection of fenestartion and dehiscence, full scan $\left(360^{\circ}\right)$ has a higher accuracy than half $\operatorname{scan}\left(180^{\circ}\right)[4]$.

Kamburoglu et al. assessed the effect of MAR algorithm with high, low and medium levels on the diagnostic accuracy for detection of buccal peri-implant and periodontal defects. They concluded that although the area under the curve and the diagnostic accuracy for detection of periodontal defects was bigger than the corresponding values for peri-implant defects, no significant difference was noted in diagnostic accuracy of observers in absence of MAR for the different levels of MAR algorithm [6]. Moreover, Kamburoglu et al. assessed the effect of the size of field of view and defect size on diagnostic accuracy for the detection of peri-implant defects and conclud- ed that the field size of view had no significant effect; however, the diagnostic accuracy was significantly higher for larger defects [1]. Similarly, Bachara et al. assessed the accuracy of the MAR algorithm of ProMax 3D and Master 3D for the detection of root fracture and found that application of the MAR algorithm in both systems significantly decreased the accuracy and maximum diagnostic accuracy belonged to ProMax 3D in absence of the MAR algorithm [7]. Dalili Kajan et al. assessed the effect of MAR algorithm on the diagnostic accuracy for the detection of vertical root fractures in teeth with post space and pin. They reported that although the sensitivity for the detection of vertical root fractures increased by using the MAR algorithm, the diagnostic accuracy for the detection of root fractures was not significantly different in the presence and absence of the MAR algorithm [17].

The value of the area under the ROC curve (mostly greater than 0.7 ) indicated that CBCT is an efficient tool for the detection of periimplant fenestration and dehiscence. Comparison of ProMax 3D and Cranex 3D CBCT systems in this study revealed that when the MAR algorithm was not used, the area under the curve and subsequently the diagnostic accuracy of ProMax 3D for detection of defects was bigger than that of Cranex 3D. However, when the MAR algorithm was enabled, the accuracy of Cranex 3D for detection of fenestration and dehiscence increased compared with ProMax 3D. It may be concluded that although the use of this algorithm decreases the diagnostic accuracy, this reduction in ProMax $3 \mathrm{D}$ was greater than that in Cranex 3D.

In the present study, the sensitivity and specificity of ProMax 3D for detection of fenestration and dehiscence were higher than 0.61. These values were higher than 0.70 for Cranex 3D, i.e. CBCT images obtained by the ProMax 3D and Cranex 3D CBCT systems can correctly detect the presence or absence of these defects by over $61 \%$ and $70 \%$, respectively. This finding was in line with that of Sheikhi 
et al. that CBCT images had a sensitivity and specificity higher than 0.79 for detection of fenestration and dehiscence [16]; although the CBCT systems used in the two studies were different.

In the present study, the use of the MAR algorithm in both CBCT systems decreased the sensitivity, specificity, PPV and NPV for detection of fenestration and dehiscence compared with no use of this algorithm. The only exception was related to the specificity; in addition, PPV of ProMax 3D for detection of fenestration was similar in with and without the MAR algorithm. This reduction in sensitivity and specificity may be due to the fact that the MAR algorithm not only eliminates the image artifacts but also removes some useful gray levels that are beneficial for diagnosis [18] that this result was in line with that of Sheikhi et al. [16] and Azevedo-Vaz et al. [2]. In the study by Sheikhi et al. [16], the values were higher when the MAR algorithm was not used. Also, Azevedo-Vaz et al. [2] reported higher sensitivity, specificity, PPV and NPV for both defects when the MAR algorithm was not applied. Moreover, similar to present results, they found that the specificity of ProMax 3D was the same in presence and absence of the MAR algorithm.

The manufacturers of CBCT scanners have developed different MAR algorithms, which has led to their increasing use in the recent years. The efficacy of these algorithms has been the topic of many investigations, reporting controversial results. The type and size of defects, type of CBCT system, and type of MAR algorithm and the experience of the radiologist are among the factors that may be responsible for the variability in results.

Considering the results of the present study and the available literature, the application of the MAR algorithm is not effective for detection of peri-implant fenestration and dehiscence. However, it may be useful for the detection of other defects. For example, Cebe et al. concluded that use of MAR algorithm significantly increased the diagnostic accuracy for the detection of proximal caries when the carious surface was in contact with composite or amalgam restorations [19].

The current study had an in vitro design, and fenestration and dehiscence defects were created in bovine rib blocks by a bur. Red dental wax was used to simulate the soft tissue. In vitro study, setting is different from the clinical one. For instance, in the clinical setting, the patient movement can aggravate the image artifacts (causing motion artifact) while this type of artifact is non-existent in vitro. On the other hand, beam attenuation in the soft tissue is different from that in red dental wax. Moreover, the defects created by bur are different in shape, size and borders with actual defects in vivo. Leung et al. [2] emphasized that naturally occurring defects develop gradually, have tapered borders and are more difficult to be detected compared with artificially created defects that have sharp borders. In the present study, the borders of the defects were tapered (beveled) to simulate the shape and form of natural defects.

Eventually, it should be noted that application of MAR algorithms is difficult and timeconsuming because image reconstruction takes more time as such. On the other hand, the techniques employed by these algorithms to eliminate the metal artifacts are different. In addition, the voxel size, field of view, signal to noise ratio, contrast, sensor quality, spatial resolution and the reconstruction algorithm can all affect the diagnostic quality of images.

\section{Conclusion}

Considering the CBCT systems used in this study and the applied imaging protocols, the application of MAR algorithm decreased the diagnostic accuracy for the detection of fenestration and dehiscence around dental implants. It may be concluded that application of MAR algorithm may improve the visual image quality, but it does not necessarily increase the diagnostic accuracy of images. 


\section{Conflict of Interest}

None

\section{References}

1. Kamburoğlu K, Murat S, Kılıç C, Yüksel S, Avsever H, Farman A, et al. Accuracy of CBCT images in the assessment of buccal marginal alveolar peri-implant defects: effect of field of view. Dentomaxillofac Radiol. 2014;43(4):20130332. doi: 10.1259/dmfr.20130332. PubMed PMID: 24645965. PubMed PMCID: PMC4082260.

2. De-Azevedo-Vaz SL, Peyneau PD, Ramirez-Sotelo LR, De Faria Vasconcelos K, Campos PSF, Haiter-Neto F. Efficacy of a cone beam computed tomography metal artifact reduction algorithm for the detection of peri-implant fenestrations and dehiscences. Oral Surg Oral Med Oral Pathol Oral Radiol. 2016;121(5):550-6. doi: 10.1016/j. 0000.2016.01.013. PubMed PMID: 27068312.

3. Leung CC, Palomo L, Griffith R, Hans MG. Accuracy and reliability of cone-beam computed tomography for measuring alveolar bone height and detecting bony dehiscences and fenestrations. Am J Orthod Dentofacial Orthop. 2010;137(4 Suppl):S109-19. doi: 10.1016/j. ajodo.2009.07.013. PubMed PMID: 20381751.

4. De-Azevedo-Vaz SL, De Faria Vasconcelos K, Neves FS, et al. Detection of periimplant fenestration and dehiscence with the use of two scan modes and the smallest voxel sizes of a cone-beam computed tomography device. Oral Surg Oral Med Oral Pathol Oral Radiol. 2013;115(1):121-7. doi: 10.1016/j.0000.2012.10.003. PubMed PMID: 23217543.

5. Schliephake H, Wichmann M, Donnerstag F, Vogt S. Imaging of periimplant bone levels of implants with buccal bone defects. Clin Oral Implants Res. 2003;14(2):193200. doi: 10.1034/j.1600-0501.2003.140209.x. PubMed PMID: 12656879.

6. Kamburoğlu K, Kolsuz E, Murat S, Eren H, Yüksel S, Paksoy C. Assessment of buccal marginal alveolar peri-implant and periodontal defects using a cone beam CT system with and without the application of metal artefact reduction mode. Dentomaxillofac Radiol. 2013;42(8):20130176. doi: 10.1259/ dmfr.20130176. PubMed PMID: 23956236. PubMed PMCID: PMC3922259.

7. Bechara B, Moore W, McMahan C, Noujeim M. Metal artefact reduction with cone beam CT: an in vitro study. Dentomaxillofac Radiol. 2012;41(3):248-53. doi: 10.1259/dmfr/80899839. PubMed PMID: 22241878. PubMed PMCID: PMC3520287

8. Schulze R, Heil U, Gro $\beta$ D, Bruellmann D, Dranischnikow E, Schwanecke U, et al. Artefacts in CBCT: a review. Dentomaxillofac Radiol. 2011;40(5):265-73. doi: 10.1259/dmfr/30642039. PubMed PMID: 21697151. PubMed PMCID: PMC3520262 .

9. Benic GI, Sancho-Puchades M, Jung RE, Deyhle $H$, Hämmerle $\mathrm{CH}$. In vitro assessment of artifacts induced by titanium dental implants in cone beam com- puted tomography. Clinical Oral Implants Research. 2013;24(4):378-83. doi: 10.1111/clr.12048. PubMed PMID: 23106603.

10. Parsa A, Ibrahim N, Hassan B, Syriopoulos K, Van Der Stelt P. Assessment of metal artefact reduction around dental titanium implants in cone beam CT. Dentomaxillofac Radiol. 2014;43(7):20140019. doi: 10.1259/ dmfr.20140019. PubMed PMID: 25135316. PubMed PMCID: PMC4170846.

11. Dave M, Davies J, Wilson R, Palmer R. A comparison of cone beam computed tomography and conventional periapical radiography at detecting peri-implant bone defects. Clinical Oral Implants Research. 2013;24(6):6718. doi: 10.1111/j.1600-0501.2012.02473.x. PubMed PMID: 22458628.

12. Mol A, Balasundaram A. In vitro cone beam computed tomography imaging of periodontal bone. Dentomaxillofac Radiol. 2008;37(6):319-24. doi: 10.1259/ dmfr/26475758. PubMed PMID: 18757716.

13. Mengel R, Kruse B, Flores-de-Jacoby L. Digital volume tomography in the diagnosis of peri-implant defects: an in vitro study on native pig mandibles. J Periodontol. 2006;77(7):1234-41. doi: 10.1902/jop.2006.050424. PubMed PMID: 16805688.

14. Barrett JF, Keat N. Artifacts in CT: recognition and avoidance. Radiographics. 2004;24(6):1679-91. doi: 10.1148/rg.246045065. PubMed PMID: 15537976.

15. Siewerdsen JH, Moseley D, Bakhtiar B, Richard S, Jaffray DA. The influence of antiscatter grids on softtissue detectability in cone-beam computed tomography with flat-panel detectors: Antiscatter grids in conebeam CT. Medical physics. 2004;31(12):3506-20. doi: 10.1118/1.1819789. PubMed PMID: 15651634.

16. Sheikhi M, Behfarnia P, Mostajabi M, Nasri N. The Efficacy of Metal Artifact Reduction (MAR) Algorithm in Cone Beam Computed Tomography on the Diagnostic Accuracy of Fenestration and Dehiscence around Dental Implants. J Periodontol. 2020;91(2):209-214. doi: 10.1002/JPER.18-0433. PubMed PMID: 31364765.

17. Dalili Kajan Z, Taramsari M, Khosravi Fard N, Khaksari F, Moghasem Hamidi F. The Efficacy of Metal Artifact Reduction Mode in Cone-Beam Computed Tomography Images on Diagnostic Accuracy of Root Fractures in Teeth with Intracanal Posts. Iran Endod J. 2018;13(1):4753. doi: 10.22037/iej.v13i1.17352. PubMed PMID: 29692835. PubMed PMCID: PMC5800441.

18. Meilinger M, Schmidgunst C, Schütz O, Lang EW. Metal artifact reduction in cone beam computed tomography using forward projected reconstruction information. $Z$ Med Phys. 2011;21(3):174-82. doi: 10.1016/j.zemedi.2011.03.002. PubMed PMID: 21530200.

19. Cebe F, Aktan AM, Ozsevik AS, Ciftci ME, Surmelioglu $\mathrm{HD}$. The effects of different restorative materials on the detection of approximal caries in cone-beam computed tomography scans with and without metal artifact reduction mode. Oral Surg Oral Med Oral Pathol Oral Radiol. 2017;123(3):392-400. doi: 10.1016/j. 0000.2016.11.008. PubMed PMID: 28111155. 